\title{
DINÂMICA DA ALIMENTAÇÃO NATURAL DE CALLINECTES DANAE SMITH (DECAPODA, PORTUNIDAE) NA LAGOA DA CONCEIÇÃO, FLORIANÓPOLIS, SANTA CATARINA, BRASIL
}

\author{
Joaquim Olinto Branco ${ }^{1,2}$ \\ José Roberto Verani ${ }^{3}$
}

\begin{abstract}
FEEding NATURAL DYNAMICS OF CALLINECTES DANAE SMITH (DECAPODA, Portunidae) From Lagoa da Conceição, Florianópolis, Santa Catarina, Brasil. From March/91 to February/92 monthly collection surveys were carried out to obtain basic information about the dynamics of feeding and trophic spectrum of $C$. danae Smith, 1869. A total of 456 males and 527 females were caught. The diet of the species was not sex related. The trophic spectrum of $C$. danae is composed by a high number of items. The 35 items were assembled in 14 categories and the feeding index was applied. Mollusca, Polychaeta and Crustacea were observed to be basic elements in the species diet, Osteichthyes and MOND appear as secondary resource whereas vegetal material were found to be of less importance.
\end{abstract}

KEY WORDS. Portunidae, Callinectes danae, feeding habits, Lagoa da Conceição

Callinectes danae Smith, 1869, conhecido como todas espécies do gênero por "siri azul" ocorre no Atlântico ocidental: Flórida, Golfo do México, América Central, Antilhas, norte da América do Sul, Brasil (Paraíba até o Rio Grande do Sul), Argentina e Chile (Melo et al. 1989). Habita estuários com fundo de lama, manguezais, fundo de cascalho coberto por algas, praias e oceanos até a profundidade de $70 \mathrm{~m}$, tolerando ampla variação de salinidade (WILLIAMS 1984).

O conhecimento dos hábitos alimentares dos Brachyura é de fundamental interesse porque a disponibilidade e a utilização do alimento desempenham papéis importantes em seus padrões de distribuição, migração e ecdise (MCLAUGHLIN \& HEBARD 1961), bem como para o sucesso do cultivo de espécies de importância econômica (WILLIAMS 1981).

A versatilidade dos Brachyura em capturar suas presas foi verificada para moluscos bivalves de importância econômica como ostras (ELNER \& LAVOIE 1983), mexilhões (ELNER 1978; SEED 1980) e outros moluscos (STEVENS et al. 1982) e, para peixes, (KNEIB 1982).

Os processos de captura e manipulação do alimento dificultam a identificação e quantificação dos itens alimentares presentes em seus estômagos (WILLIAMS 1981; STEVENS et al. 1982; HAEFNER 1990). A atividade alimentar dos Brachyura

1) Faculdade de Ciências do Mar, Universidade Vale do Itajai. Caixa Postal $360,88301-970$ Itajai, Santa Catarina, Brasil.

2) Núcleo de Estudos do Mar, Centro de Ciências Biológicas, Universidade Federal de Santa Catarina. Caixa Postal 474, 88040-900, Florianópolis, Santa Catarina, Brasil.

3) Universidade Federal de São Carlos. Caixa Postal 676, 13565-905 São Carlos, São Paulo, Brasil. 
pode ser dividida em: captura do alimento através dos quelípodes, trituração pelas peças bucais e passagem do alimento da boca ao estômago e quebra mecânica do alimento pelos ossículos do estômago e digestão química. Dessa maneira, ao analisarmos a dieta de um siri, o alimento já passou no mínimo pelas duas etapas iniciais.

O estudo da dieta natural de Crustacea Decapoda Brachyura tem sido muito discutida mas, com exceção de WiLliams (1981), não existem trabalhos que abordem especificamente o assunto. RoPES (1968) e STEVENS et al. (1982) fazem um alerta sobre as limitações existentes nos diferentes métodos, sempre embasados naqueles utilizados em estudos de alimentação de peixes.

BRANCO \& MASUNARI (1992) estudaram a estrutura populacional de $C$. danae na lagoa, abordando, aspectos do ciclo de vida, migração e relação peso/largura da carapaça; fecundidade (BRANCO \& AVILA 1992), período de desova (BRANCO et al. (1992), crescimento (BRANCO \& MASUNARI 1992b). Em continuidade ao estudo da biologia e ecologia de $C$. danae, propõe-se neste trabalho o conhecimento do hábito alimentar, suas variações sazonais através da análise do conteúdo estomacal.

\section{MATERIAL E MÉTODOS}

As coletas foram realizadas mensalmente no canal da Lagoa da Conceição durante o período de março/91 a fevereiro/92 (BRANCO 1996a).

Os exemplares foram coletados com o auxílio de seis jererés, com $50 \mathrm{~cm}$ de diâmetro, malha de 10 milímetros entre nós e cabo de nylon com $15 \mathrm{~m}$ de comprimento. Em cada armadilha foi colocada isca de peixe fresco, protegida em saco de nylon duplo (tela tipo mosquiteiro) para evitar a ingestão das iscas peloś siris. Os exemplares coletados foram mantidos em "freezer" até serem trabalhados.

A identificação e reconhecimento do sexo foram realizados de acordo com Williams (1974), e os estádios de maturação (jovem/adulto) foram determinados pelo formato e aderência do abdome aos esternitos torácicos, conforme TAISSOUN (1969). Sempre que necessário recorreu-se ao auxílio de microscópio estereoscópio.

A largura da carapaça (largura entre as pontas dos espinhos laterais) foi medida com ictiômetro graduado em milímetros e o peso do corpo em balança com precisão de centésimos de grama.

\section{Análise do conteúdo estomacal}

Foram utilizados apenas exemplares em intermudas, sendo rejeitados os indivíduos em ecdise de acordo com o sugerido por HAEFNER (1990). Os estômagos foram retirados após a remoção da carapaça com auxílio de tesoura e pinça. Os estômagos foram inicialmente pesados, abertos e estimado o grau de repleção estomacal determinado de acordo com a quantidade de alimento presente nos estômagos.

Em seguida, o estômago foi pesado, o conteúdo estomacal foi removido com jatos de água (frasco lavador) e depositado em placa de Petri com água. O estômago foi secado em papel absorvente e pesado. 
A identificação dos itens alimentares foi realizada conforme Rios (1975), NonAto \& AmAral (1979), Amaral \& NonATO (1981, 1982), BARNES (1984), ELNER et al. (1985) e NONATO et al. (1990). Os ítens do conteúdo que não puderam ser identificados, devido ao elevado grau de digestão foram considerados como Matéria Orgânica Não Determinada (MOND).

\section{Análise dos dados}

Considerando que os siris raramente ingerem suas presas íntegras e que, na maioria das vezes, manipulam seu alimento com as quelas e mandíbulas, consumindo apenas parte da mesma, o número de organismos ingeridos não constitui o elemento principal na avaliação da alimentação natural de $C$. danae.

Assim, optou-se por utilizar o método dos pontos e o de freqüência de ocorrência, conforme sugerido por WILLIAMS (1981), WEAR \& HADDON (1987) e HAEFNER (1990), complementado pelo índice alimentar (KAWAKAMI \& VAZZOLER 1980), o que contribui para a compreensão global da dieta de C. danae, evidenciando aspectos que podem influenciá-la e tornar possíveis comparações com outras espécies, facilitando o entendimento da relação predador-presa.

Para verificar a possível ocorrência de diferenças significativas entre a freqüência relativa de alimento consumido por sexo, foi utilizado o teste do $X^{2}$ (qui-quadrado) (LuDWIG \& REYNOLDS 1988) a nível de 5\% de significância e n-1 graus de liberdade $(\mathrm{n}=2)$.

Os estádios de repleção foram baseados na quantidade relativa de alimento presente nos estômagos: (I) vazio, (II) médio, (III) cheio. O grau médio de repleção (Gr) foi calculado, conforme SANTOS (1978):

$$
G r=\frac{\sum i \cdot f a}{\sum f a}
$$

onde: (i) grau de repleção quantificado, (fa) freqüência absoluta do grau "i" de repleção.

O índice de repleção médio (Ir) foi estimado de acordo com SANTOS (1978) e BARBIERI et al. (1982):

$$
I r=\frac{w e}{w t} \rightarrow I r=\frac{\sum I r}{n}
$$

onde: (we) peso do estômago, (wt) peso do indivíduo, (n) número de exemplares.

A relação peso/largura da carapaça e o fator de condição foram calculados de acordo com SANTOS (1978):

$$
W t=\phi w i d^{\theta}
$$

onde: $(\phi)$ fator de condição, $(\theta)$ constante, relacionada com o tipo de crescimento do siri.

Nas análises quali-quantitativa, foram utilizados os métodos de freqüência de ocorrência (FO) e dos pontos (MP) conforme HyNES (1950) e WiLLIAMS (1981). 
Freqüência de ocorrência (FO)

$$
F O=\frac{b i}{N} \cdot 100
$$

onde: (bi) número de estômagos que contém o item i, (N) número de exemplares amostrados.

\section{Método dos pontos (MP)}

Este método foi aplicado seguindo a metodologia utilizada por WiLliams (1981) e WEAR \& HADDON (1987). A contribuição relativa de cada item, no volume total de alimento em um determinado estômago, foi subjetivamente determinada em uma escala de pontos com cinco graus: 1 ) $<5 \%=2,5$ pontos, 2 ) $5-35 \%=25$ pontos, 3) $35-65 \%=50$ pontos, 4) $65-95 \%=75$ pontos, 5) $>95 \%=100$ pontos.

O número de pontos que cada item recebeu foi atribuído conforme o grau de repleção.

A percentagem total de pontos para um item foi expressa pela fórmula (WILliams 1981):

$$
\sum_{j=1}^{n}\left(\frac{a i j}{A}\right) .100
$$

onde: (A) número total de pontos para todos os itens, (n) número total de estômagos examinados; (aij) número de pontos do item presa "i" encontrado nos estômagos dos exemplares examinados.

A análise quantitativa dos itens alimentares foi baseada em categorias tróficas (grandes grupos taxonômicos) considerando-se sua freqüência de ocorrência e pontos (volume relativo). Para efeito de comparação e de melhor compreensão da importância de cada categoria trófica foi utilizado o índice alimentar proposto por KAWAKAMI \& VAZZOLER (1980) que combina os dois métodos, calculado de acordo com a fórmula:

$$
I A i=\frac{F A \cdot M P}{\sum_{i=1}^{n}(F A \cdot M P)}
$$

onde: (IAi) Índice alimentar, $(\mathrm{i}=1,2 \ldots . ., \mathrm{n})$ determinado item alimentar; (FA) Freqüência de ocorrência do item "i" (\%); (MP) pontos do item "i" (\%).

\section{RESUL.TADOS}

\section{Dinâmica da alimentação}

Durante o período março/91 a fevereiro/92 foram analisados 983 estômagos de $C$. danae; destes, 218 (22,2 \%) estavam vazios e 765 estômagos (77,8 \%) apresentaram conteúdo. Dos 456 machos examinados, 91 (20,0 \%) mostraram estômago vazio e $365(80,0$ \%) continham algum tipo de alimento, das 527 fêmeas analisadas, $127(24,1 \%)$ apresentaram estômago vazio e 400 (75,9\%) com alimento (Tab. I). 
A freqüência de exemplares com estômago vazio foi acentuadamente mais baixa do que aqueles com conteúdo estomacal em ambos os sexos (Tab. I).

Tab. I. Callinectes danae. Distribuição de freqüência de ocorrência de machos e fêmeas com estômago vazio ou com conteúdo durante o período de coleta.

\begin{tabular}{|c|c|c|c|c|c|}
\hline \multirow{2}{*}{ Sexo } & \multicolumn{2}{|c|}{ Estômagos vazios } & \multicolumn{2}{|c|}{ Estômagos com conteúdo } & \multirow{2}{*}{ Total } \\
\hline & $\mathrm{n}$ & $\%$ & $\mathrm{n}$ & $\%$ & \\
\hline Machos & 91 & 20,0 & 365 & 80,0 & 456 \\
\hline Fêmeas & 127 & 24,1 & 400 & 75,9 & 527 \\
\hline
\end{tabular}

$\mathrm{O}$ teste do $X^{2}(\mathrm{P} \leq 0,05)$ aplicado à freqüência percentual relativa em pontos das 14 categorias de presas, material não identificado e areia não apresentou diferença significativa na proporção dos itens alimentares consumidos por machos e fềmeas (Tab. II). Assim, a dieta natural da população foi analisada para sexos grupados.

Tab. II. Callinectes danae. Frequência absoluta de pontos (MP) e freqüência percentual (\%) dos taxa das presas, detritos e areia na dieta natural e o teste do $X^{2}$ entre as freqüências relativas para machos e fêmeas durante o período de março/91 a fevereiro/92.

\begin{tabular}{|c|c|c|c|c|c|}
\hline \multirow{2}{*}{ Categorias alimentares } & \multicolumn{2}{|c|}{ Machos } & \multicolumn{2}{|c|}{ Fêmeas } & \multirow{2}{*}{$x^{2}$} \\
\hline & MP & $\%$ & MP & $\%$ & \\
\hline Algas & 487 & 3,30 & 302 & 1,80 & 0,4 \\
\hline Macrófitas & 166 & 1,10 & 355 & 2,20 & 0,3 \\
\hline Foraminiferida & - & - & 2 & 0,01 & - \\
\hline Porifera & 2 & 0,01 & - & - & - \\
\hline Cnidaria & 7 & 0,004 & 28 & 0,20 & 0,1 \\
\hline Mollusca & 2137 & 14,80 & 2383 & 14,60 & 0,0 \\
\hline Polychaeta & 3509 & 24,20 & 4052 & 24,80 & 0,0 \\
\hline Crustacea & 3633 & 25,10 & 3101 & 18,90 & 0,8 \\
\hline Insecta & 8 & 0,05 & 8 & 0,05 & 0,0 \\
\hline Echinodermata & 4 & 0,02 & 6 & 0,03 & 0,0 \\
\hline Ascidiacea & 2 & 0,01 & 2 & 0,01 & 0,0 \\
\hline Osteichthyes & 1782 & 12,30 & 3119 & 19,10 & 1,4 \\
\hline MOND & 503 & 3,50 & 437 & 2,60 & 0,1 \\
\hline Areia & 2268 & 15,60 & 2573 & 15,70 & 0,0 \\
\hline Total & 14508 & 100,00 & 16368 & 100,00 & \\
\hline
\end{tabular}

*. Significativo $P \leq 0,05$.

As relações lineares existentes entre o peso médio dos estômagos e peso médio total dos siris, por estádio de repleção (Fig. 1), comprovadas analiticamente pelo valor dos coeficientes de correlação linear de Pearson (variando de 0,9135 a 0,9856 ), permitiu estimar os valores médios do grau e do índice de repleção (Fig. $2 a, b)$.

A figura 2a apresenta a variação mensal do grau médio de repleção dos estômagos. O valor mais elevado foi registrado em março/91, decrescendo gradativamente até atingir o menor valor em dezembro/91, recuperando-se a partir de 


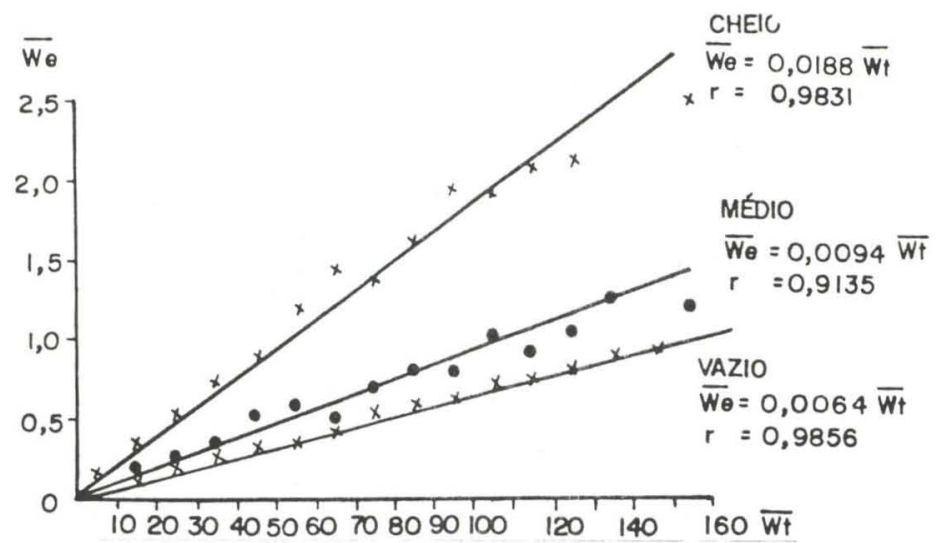

Fig. 1. Callinectes danae. Relação entre o peso médio do corpo e o peso médio do estômago por estádio de repleção.
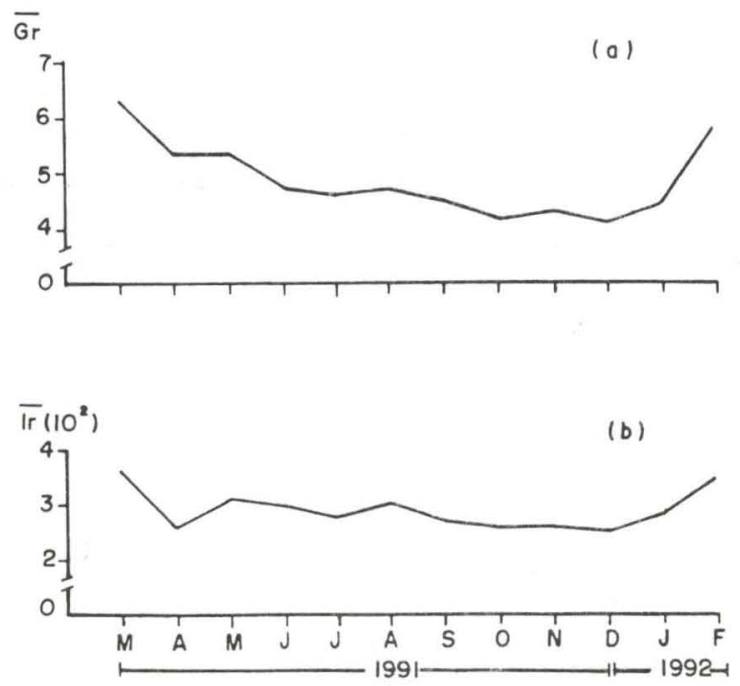

Fig. 2. Callinectes danae. Variação mensal do grau médio (a) e do índice médio de repleção (b) dos estômagos durante o periodo de março/91 a fevereiro/92.

janeiro/92. O índice médio de repleção (Fig. 2b) apresenta a mesma tendência de variação do grau médio de repleção. Analisando-se a figura $2 \mathrm{a}$ e $2 \mathrm{~b}$ por estação do ano, observa-se que a partir do início do outono ocorre uma redução gradual na ingestão de alimento atingindo os menores valores na primavera.

A equação $W t=\phi$ wid $^{\theta}$, mostrou ser válida para a população de $C$. danae através da dispersão dos valores do peso total (Wt) lançados em gráfico, em funçãc da largura da carapaça (Wid). A amplitude de variação da largura da carapaça na população foi de 2,7 a $13,8 \mathrm{~cm}$ e do peso foi de 1,0 a $151,5 \mathrm{~g}$. O logarítmo destas variáveis mostrou uma relação linear, segundo a expressão (Fig. 3):

$\ln W \mathrm{t}=-2,6831+2,9491 \ln \mathrm{Wid}$, sendo $\mathrm{r}=0,9798$. 


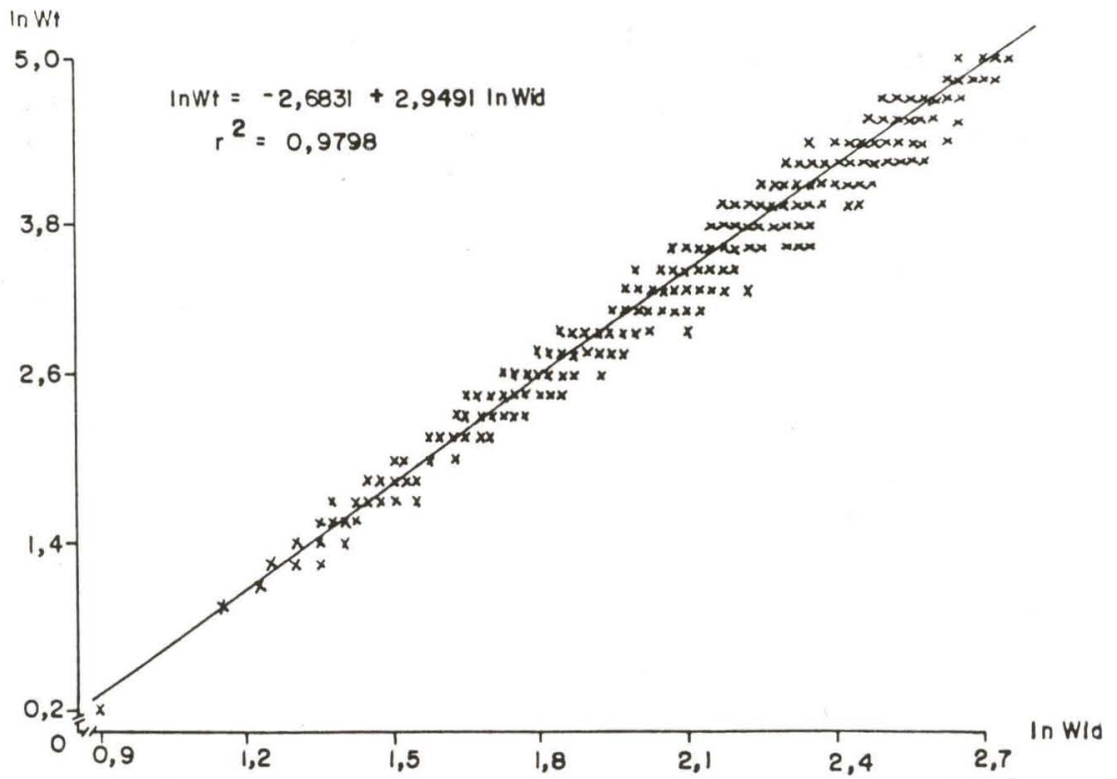

Fig. 3. Callinectes danae. Transformação logarítmica da relação peso/largura da carapaça.

A curva de flutuação dos valores médios do fator de condição apresentou dois picos durante o ano: o primeiro em junho/91 e o segundo em janeiro/92 (Fig. 4). O peso do estômago exerce pequena influência na variação do fator de condição. Os valores do fator de condição variaram entre 0,0660 (março/91) e 0,0731 (janeiro/92), enquanto que, sem a influência do peso do estômago variaram entre 0,0651 (março/91 e maio/91) e 0,0720 (janeiro/92). A variação dos valores médios do fator de condição não está diretamente relacionada com a variação do grau e do índice médio de repleção dos estômagos, visto que os valores médios mais elevados ocorreram em períodos diferentes.

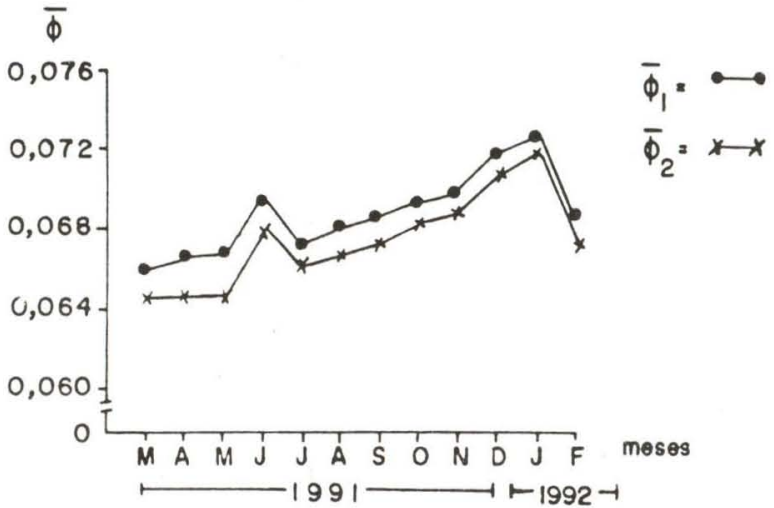

Fig. 4. Callınectes aanae. Variaçăo média mensal do fator de condiçăo total $\left(\phi_{1}\right)$ e sem a influência do peso do estômago ( $\left.\phi_{2}\right)$. 


\section{Espectro trófico}

Através da análise do conteúdo estomacal dos exemplares foi possível identificar 35 itens componentes da dieta natural de $C$. danae (Tab. III). Embora, areia faça parte dessa relação, não foi utilizada nas análises. Assim, os itens mais importantes em freqüência de ocorrência e pontos foram Anomalocardia brasiliana (Gmelin, 1791), Brachyura, Polychaeta não idenficado, MOND, Osteichthyes, Polynoidae, macrófitas, ovos de Crustacea, Bulla striata Bruquière, 1792 e algas. Os menos frequientes foram Foraminiferida, Porifera, Caprellidae, Diptera, Ostracoda, e Ascidiacea.

Tab. III. Callinectes danae. Freqüência de ocorrência (FO) e de pontos (MP) dos itens alimentares no conteúdo estomacal durante o periodo de março/91 a fevereiro/92.

\begin{tabular}{|c|c|c|}
\hline Itens & $\mathrm{FO}$ & MP \\
\hline Algas & 48 & 789 \\
\hline Macrófitas & 106 & 521 \\
\hline Foraminiferida & 1 & 2 \\
\hline Porifera & 1 & 2 \\
\hline Cnidaria & 6 & 35 \\
\hline \multicolumn{3}{|l|}{ Mollusca } \\
\hline Perna perna (Linnaeus, 1758) & 8 & 100 \\
\hline Brachidontes darwinianus (Orbigny, 1846) & 32 & 402 \\
\hline Anomalocardia brasiliana (Gmelin, 1791) & 259 & 3257 \\
\hline Massarius vibex (Say, 1822) & 8 & 16 \\
\hline Bulla striata Bruquiere, 1792 & 63 & 619 \\
\hline Littoridina australis (Orbigny, 1835) & 8 & 85 \\
\hline Mollusca não identificados & 14 & 141 \\
\hline \multicolumn{3}{|l|}{ Polychaeta } \\
\hline Polynoidae & 115 & 2736 \\
\hline Nereidae & 65 & 1170 \\
\hline Eunicidae & 10 & 231 \\
\hline Maldanidae & 8 & 527 \\
\hline Pectinaridae & 14 & 254 \\
\hline Polychaeta não identificados & 162 & 2543 \\
\hline \multicolumn{3}{|l|}{ Crustacea } \\
\hline Ostracoda & 2 & 65 \\
\hline Cirripedia & 33 & 488 \\
\hline Caprellidae & 1 & 2 \\
\hline Penaeidae & 9 & 110 \\
\hline Callinectes spp. & 39 & 1114 \\
\hline Xanthidae & 3 & 6 \\
\hline Grapsidae & 13 & 354 \\
\hline Brachyura näo identificados & 186 & 3290 \\
\hline Ovos de Crustacea & 67 & 1305 \\
\hline \multicolumn{3}{|l|}{ Insecta } \\
\hline Coleoptera & 12 & 24 \\
\hline Lepidoptera & 3 & 6 \\
\hline Diptera & 1 & 2 \\
\hline Echinodermata & 5 & 10 \\
\hline Ascidiacea & 2 & 4 \\
\hline Osteichthyes & 132 & 4901 \\
\hline MOND & 157 & 941 \\
\hline Areia & 634 & 4824 \\
\hline Total & 2227 & 30876 \\
\hline
\end{tabular}




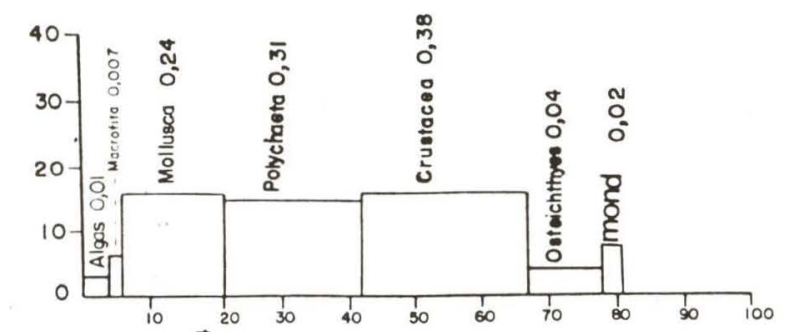

PRIMAVERA

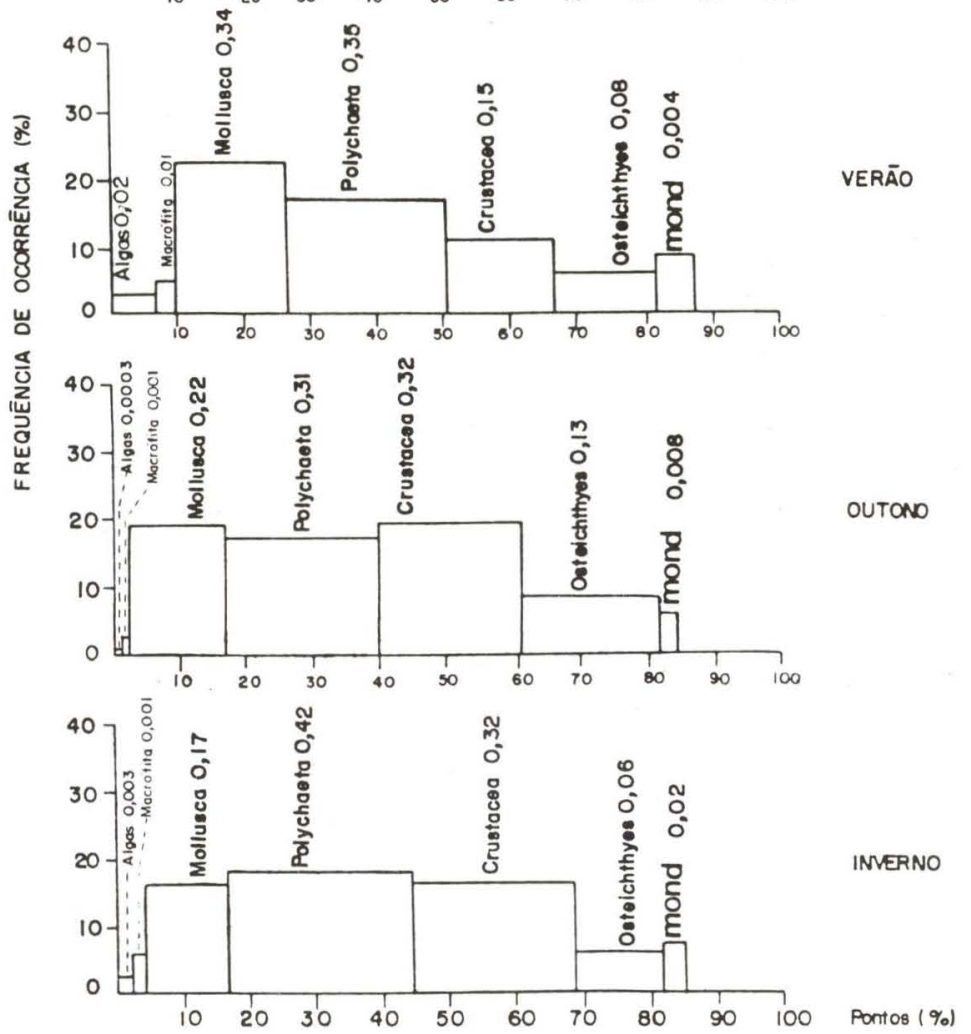

Fig. 5. Callinectes danae. Freqüëncia de ocorrência (\%) e de pontos (\%) uos grupos alımentares e seus respectivos indices alimentares, por estação do ano.

Os 35 itens foram agrupados em 14 categorias (Tab. II). Os métodos de análise empregados indicam que os Mollusca, Polychaeta e Crustacea, destacam-se como elementos básicos na dieta de $C$. danae. Osteichthyes e MOND aparecem como recurso secundário. Matéria vegetal caracteriza-se como recurso terciário, enquanto que outros componentes da dieta de $C$. danae, sem influência da areia foram considerados como acidentais na dieta (Tab. III).

Apesar da areia representar fração significativa no cômputo geral, tanto em ocorrência como em pontos, provavelmente a ingestão da mesma ocorra juntamente com as presas. 
Analisando o regime alimentar de C. danae, de acordo com o IAi, por estação do ano (Fig. 5), verifica-se que ocorrem flutuações sazonais dos principais grupos. Na primavera, a contribuição em termos de freqüência de ocorrência dos Mollusca, Polychaeta e Crustacea são praticamente equivalentes, embora os Crustacea contribuam com maior porcentagem de pontos, seguidos dos Polychaeta, Mollusca, Osteichthyes, MOND, algas e macrófitas. Os valores de IA i corroboram os resultados confirmando a importância dos Crustacea $(0,38)$, Polychaeta $(0,31)$, Mollusca $(0,24)$ e Osteichthyes $(0,04)$ na dieta da espécie (Fig. 5).

No verão, os Mollusca dominaram em freqüência de ocorrência, seguidos dos Polychaeta, Crustacea, material não identificado, Osteichthyes, macrófitas e algas. Em freqüência de pontos, os Polychaeta foram o recurso mais explorado, enquanto que Mollusca e Crustacea contribuíram em proporções semelhantes. $\mathrm{O}$ IAi confirma os Polychaeta $(0,35)$ como o principal recurso alimentar, seguido dos Mollusca $(0,34)$, Crustacea $(0,15)$, Osteichthyes $(0,08)$, MOND $(0,04)$, algas $(0,02)$ e macrófitas $(0,01)$ (Fig. 5).

No outono, os Crustacea foram predados com maior freqüência, seguidos dos Mollusca, Polychaeta e Osteichthyes. Em freqüência de pontos, os Polychaeta dominaram, os Crustacea aparecem como segundo recurso, enquanto que os Osteichthyes ultrapassam os Mollusca. O IAi indica que os Crustacea foram o principal recurso alimentar explorado, seguido dos Polychaeta, Mollusca e Osteichthyes. MOND e algas foram pouco utilizados nesta estação (Fig. 5).

No inverno, os Polychaeta foram o principal componente em frequeência de ocorrência, freqüência de pontos e com IAi $(0,42)$, seguidos de Crustacea $(0,32)$, Mollusca $(0,17)$, Osteichthyes $(0,06)$, MOND $(0,02)$, macrófitas $(0,01)$ e algas $(0,003)$ (Fig. 5).

A figura 6 apresenta a contribuição em freqüência de ocorrência, pontos e o índice alimentar. Os Mollusca foram os componentes mais freqüentes na dieta de C. danae, enquanto que, Polychaeta, Crustacea e Osteichthyes dominaram em pontos. De acordo com IAi, os Polychaeta $(0,35)$, Crustacea $(0,30)$ e Mollusca $(0,23)$ formam o principal grupo, Osteichthyes $(0,08)$ e MOND $(0,01)$ o segundo e macrófitas $(0,006)$ e algas $(0,004)$ o terceiro. Foraminiferida, Porifera, Cnidaria, Insecta, Echinodermata e Ascidiacea, agrupados como outros $(0,0001)$, foram considerados de ocorrência acidental na dieta da espécie. Estes resultados são corroborados pela análise da Tab. III.

\section{DISCUSSÃO}

Neste estudo, o teste do $X^{2}$ não resultou em diferença significativa entre o volume relativo de alimento consumido por machos e fêmeas de $C$. danae. Comportamento semelhante foi registrado por WEAR \& HADDON (1987) para a população de Ovalipes catharus (White, 1843) da Nova Zelândia e por LAUGHLIN (1982) para C. sapidus Rathbun, 1896 do Estuário de Apalachicola, Flórida (EUA).

A relação linear pela origem entre o peso médio dos estômagos e peso médio dos siris, corroborado pelo alto valor do coeficiente de correlação linear de Pearson (r), permitiu enquadrar o estômago em três estádios de repleção. O estádio médio 
de repleção não manteve a tendência central, inicialmente observada, em função dos diferentes graus de digestão que influenciaram o peso médio dos estômagos. Essa tendência pode ser associada a espécies com período irregular de alimentação.

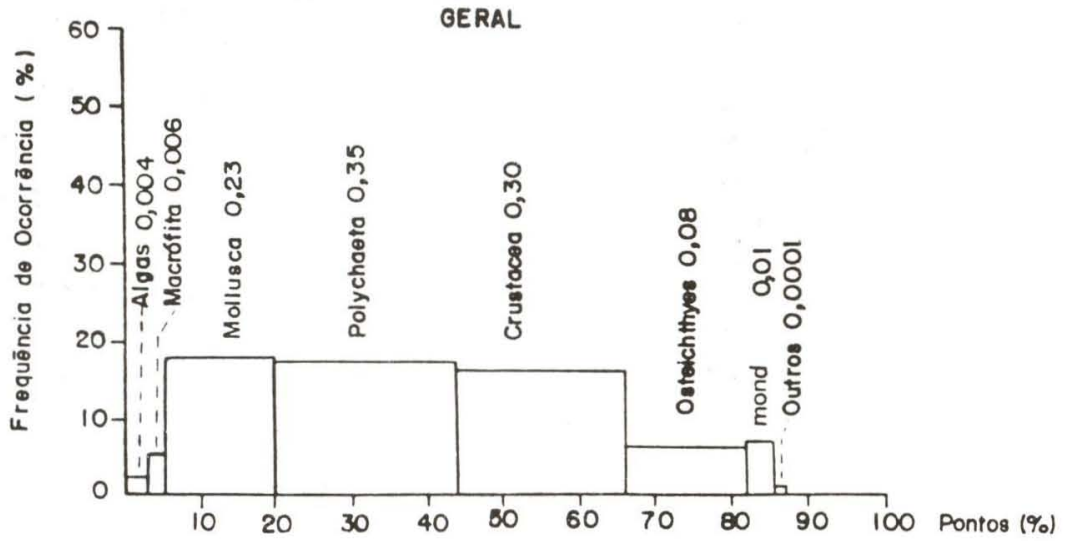

Fig. 6. Callinectes danae. Frequencıa de ocorrência (\%) e pontos (\%) e seus respectıvos indices alimentares (outros = Porifera, Foraminiferida, Cnidaria, Echinodermata e Ascidiacea) .

$\mathrm{O}$ índice médio mensal de repleção dos estômagos de $C$. danae apresentou o mesmo padrão de variação do grau médio. A literatura disponível sobre hábitos alimentares dos Brachyura não contempla esse assunto. Para peixes, BARBIERI et al. (1982) encontraram a mesma relação entre o índice médio e grau médio de repleção dos estômagos de Hoplias malabaricus (Bloch, 1794 da represa do Lobo, São Paulo.

O valor de $\theta$ da relação peso/largura está associado ao crescimento relativo entre as duas variáveis, sendo a isometria na forma do corpo fixada no valor de 3,0 (SANTOS 1978). Assim, o valor de $\theta=2,9491$ estimado neste estudo, indica que $C$. danae apresenta crescimento com fraca tendência à alometria negativa, sendo próximo ao valor registrado por BRANCO \& MASUNARI (1992a).

Segundo LE CREN (1951) o fator de condição, em geral, tem sido determinado como a razão entre o peso total do exemplar e o cubo do seu comprimento, podendo variar em função do teor de gordura, adaptação ao meio ambiente ou desenvolvimento das gônadas. Aparentemente, o fator de condição não está diretamente relacionado com o grau de repleção dos estômagos, visto que os valores médios mais elevados antecedem os períodos de maior incremento do índice médio de repleção. Entretanto a diferença entre os valores médios do fator de condição total e os valores sem influência do peso dos estômagos ocorre a partir da segunda casa decimal. Assim, as variações no fator de condição podem estar mais relacionadas ao desenvolvimento gonadal, que caracteriza um ciclo reprodutivo. Para BRANCO et al. (1992) o extenso ciclo reprodutivo de $C$. danae faz com que o peso das gônadas exerça maior influência no fator de condição que o peso dos estômagos.

BosCHI \& ANGELESCU (1962) associam as grandes diferenças no fator de condição de Hymenopenaeus mulleri [= Pleoticus muelleri (Bate, 1888)] com as 
variações do peso das gônadas durante o período reprodutivo. Comportamento semelhante foi registrado por BRANCO \& MASUNARI (1992a) para C. danae.

Um aspecto importante sobre a metodologia empregada diz respeito à análise qualitativa do conteúdo estomacal. Foi possível a identificação de 35 itens componentes da dieta de $C$. danae. Alguns a nível específico quando foram encontrados nos estômagos estruturas características das espécies mas, em grande parte dos casos, o reconhecimento das presas se restringiu a grupos familiares. Estas constatações são comuns em trabalhos sobre alimentação natural de Brachyura. Entretanto, alguns autores dispõem de um levantamento faunístico completo da região estudada, ampliando as possibilidades de identificação a nível de espécie. Segundo BRANCO (1996b), a identificação a nível específico não é o mais importante e sim determinar o espectro alimentar das espécies e a relação entre elas.

A contribuição relativa em pontos e ocorrência das categorias na dieta de $C$. danae da Lagoa da Conceição apresentou resultados similares. Em alguns casos um dos métodos levou à superestimativa da importância de um determinado item, como areia e Osteichthyes, mas não chegando a alterar as categorias dominantes que foram: Polychaeta, Crustacea, Mollusca, Osteichthyes, MOND e matéria vegetal. Diferenças observadas na dieta dos Portunidae, acima mencionadas, provavelmente estão associadas à distribuição e abundância das presas nas regiões temperadas e tropicais.

A ingestão de areia por $C$. danae deve ocorrer juntamente com as presas, o que reforçaria a hipótese de ingestão acidental. Entretanto, existe a possibilidade de ser voluntária, se considerarmos que o sedimento marinho é colonizado por uma variedade de microrganismos, tais como Ostracoda e Foraminiferida, considerados presas de baixo valor energético. Vários autores registram a ocorrência de areia nos estômagos dos Brachyura analisados, mas não a consideram como um componente alimentar. HAEFNER (1990) considerou a areia como uma fonte de carbonato e o item mais freqüente na dieta de $C$. ornatus.

O predomínio dos Mollusca na dieta dos Brachyura foi registrado por vários autores: ROPES (1968), LAUGHLIN (1982), WEAR \& HADDON (1987), HAEFNER (1990), dentre outros. PAUL (1981) registrou o predomínio de Crustacea. Segundo WiLliams (1982) os Mollusca são um alimento importante em todos os habitats, entretanto, quando disponiveis, os Crustacea são de primeira importância como alimento.

Os Polychaeta demonstram ser um componente alimentar muito importante na dieta dos Brachyura, ocorrendo em aproximadamente 30 \% dos estômagos, sendo um elo vital na cadeia trófica da região de Ubatuba, SP (NONATO et al. 1990).

A presença de material de origem vegetal nos estômagos dos Brachyura, tipicamente carnivoros, pode estar associada à ingestão acidental. Embora $C$. sapidus tenha sido observado comendo macrófitas vivas (DARNELI 1958), não existem informações se espécies de Callinectes Stimpson, 1860 podem digerir vegetais. Para PAUL (1981) permanece a dúvida sobre a presença de matéria vegetal nos estômagos de C. arcuatus Ordway, 1863 e C. toxotes Ordway, 1863, quanto à ingestão seletiva, acidental ou oriunda do trato digestivo das presas consumidas. Já em exemplares adultos de Liocarcinus puber, algas marrom (Laminaria e Fucus) 
ocorreram em 80 \% dos estômagos examinados, representando mais de $40 \%$ do volume total da dieta (CHOY 1986).

Neste estudo, algas e macrófita contribuíram em conjunto na dieta de $C$. danae com $6,9 \%$ em ocorrência e $4,2 \%$ em pontos, o que reforça a possibilidade de ingestão seletiva. Por outro lado, algas são conhecidas como suporte para uma fauna variada de Cnidaria-Hydrozoa, Mollusca, Polychaeta, Crustacea e Bryozoa (SEED et al. 1981), presas, em sua maioria, encontradas também na dieta de C. danae.

Embora os peixes sejam um recurso abundante na Lagoa da Conceição e comumente consumidos pelos siris, restam dúvidas se $C$, danae pode abater peixes de grande porte. É provável que a maioria dos Osteichthyes consumidos resulte do descarte da pesca de camarão ou de atividade saprófaga. PAUL (1981) sugere que os peixes remanescestes nos estômagos de C. arcuatus resultem da ingestão de peixes mortos descartados pelos pescadores. BRANCO (1996b) afirma que os peixes foram freqüentes na dieta dos juvenis de $C$. danae, sugerindo que haja saprofagia de restos de peixes mortos.

O canibalismo e predação interespecifica entre os Brachyura é comum na natureza, principalmente sobre juvenis, nos indivíduos em ecdise ou naqueles doentes (PAUL 1981; ELNER 1981; Williams 1982). Callinectes spp. juntamente com outros Brachyura, constituem um componente importante na dieta de $C$. danae.

C. danae é quase que exclusivamente carnívoro, sendo que material de origem vegetal é raro em sua dieta. Macroinvertebrados, especialmente anelídeos Polychaeta, Crustacea Brachyura, Mollusca bivalves e gastrópodos, asseguram a manutenção da espécie na Lagoa da Conceição. Resultados similares foram obtidos para outras espécies de Callinectes (LAUGHLIN 1982; LIPCIUS \& HINES 1986; HAEFNER 1990; STONER \& BUCHANAN 1990).

Variações sazonais na dieta de $C$. danae refletem variações na disponibilidade das espécies de presas durante o ano (BRANCO 1996b). Os Crustacea foram o principal componente, em termos de índice alimentar na primavera e outono, já os Polychaeta assumem maior importância no verão e inverno. Para PAUL (1981), LAUGHLIN (1982), e LAZARO-CHAVEZ \& BUCKLE-RAMIREZ (1994), variações sazonais na dieta de Callinectes spp. ocorrem em função da estação do ano e disponibilidade das presas.

Enquanto nenhum método quantitativo seja ideal para avaliar a dieta dos Brachyura, o índice alimentar (IAi) de KAWAKAMI \& VAZZOLER (1980) mostrou-se adequado para $C$. danae, uma vez que combina dois métodos, freqüência de ocorrência e pontos, largamente aceitos.

\section{REFERÊNCIAS BIBLIOGRÁFICAS}

AMARAL, A.C.Z. \& E.F. NONATO. 1981. Anelídeos poliquetas da costa brasileira; características e chave para famílias; glossário. Brasília, CNPq., Vol. 1/2, $47 \mathrm{p}$.

1982. Anelídeos poliquetas da costa brasileira: Aphroditidae e Polynoidae. Brasília, CNPq, Vol. 3, 46p.

BARNES, R.D. 1984. Zoologia dos Invertebrados. São Paulo, Livraria Roca, 


\section{$1179 \mathrm{p}$.}

BARBIERI, G; J.R. VERANI \& M.C. BARBIERI. 1982. Dinâmica quantitativa da nutrição de Hoplias malabaricus (Bloch, 1794), na Represa do Lobo, BrotasItirapina, SP (Pisces, Erythrinidae). Rev. Brasil. Biol. 42 (2): 295-302.

BosCHI, E.E. \& V. ANgELESCU. 1962. Description de la morfologia externa y interna del lagostino com algunas aplicaciones de indole taxonómica y biológica Hymenopenaeus mulleri (Bate) Crustacea, fam. Penaeidae. Mar del Plata. Inst. Biol. mar. 1: 5-74.

BRANCO, J.O. 1996a. Ciclo e ritmo alimentar de Callinectes danae Smith, 1869 (Decapoda, Portunidae) na Lagoa da Conceição, Florianópolis, SC. Arq. Biol. Tecnol., Curitiba, 39 (4): 987-998.

1996b. Variações sazonais e ontogênicas na dieta natural de Callinectes danae Smith 1869 (Decapoda, Portunidae) na Lagoa da Conceição, Florianópolis, SC. Arq. Biol. Tecnol., Curitiba, 39 (4): 999-1012.

BRANCO, J.O. \& S. MASUNARI. 1992a. Estrutura populacional de Callinectes danae (Decapoda, Portunidae) da Lagoa da Conceição, Florianópolis, SC, Brasil. Acta Biol. Par., Curitiba, 21 (1/4): 37-56.

Branco, J.O. \& M.G. AvilA. 1992. Fecundidade em Callinectes danae Smith (Decapoda, Portunidade) da Lagoa da Conceição, Florianópolis, Santa Catarina, Brasil. Revta bras. Zool. 9 (3/4): 167-173.

Branco, J.O.; M.J. Lunardon; M.G. Avila \& C.F. Miguez. 1992. Condição e índice gonadossomático como indicadores do período de desova em Callinectes danae Smith (Crustacea, Portunidae) da Lagoa da Conceição, Santa Catarina, Brasil. Revta bras. Zool. 9 (3/4): 175-180.

Branco, J.O. \& S. MASUNARI. 1992b. Crescimento de Callinectes danae Smith (Decapoda, Portunidae) da Lagoa da Conceição, Florianópolis, Santa Catarina, Brasil. Revta bras. Zool. 9 (1/2): 53-66.

CHOY, S.C. 1986. Natural diet and feeding habits of the crabs Liocarcinus puber and L. holsatus (Decapoda, Brachyura, Portunidae). Mar. Ecol. Prog. Ser. 31: 87-99.

DARNELL, R.M. 1958. Food habits of fishes and larger invertebrates of Lake Pontchartrain, Louisiana, an estuarine community. Publs Inst. Mar. Sci. Univ. Tex. 5: 353-416.

ELNER, R.W. 1978. The mechanics of predation by the shore crab, Carcinus maenas (L.), on the edible mussel, Mytilus edulis L. Oecologia 36 (3): 333-344.

ELNER, R.W. 1981. Diet of green crab Carcinus maenas (L) from Port Hebert, southwestern Nova Scotia. Jour. Shellfish Res. 1: 89-94.

Elner, R.W; P.G. Beninger; L.E. Linkletter \& S. LANTEigne. 1985. Guide to indicator fragments of principal prey taxa in the stomachs of two common Atlantic crab species: Cancer borealis Stimpson, 1859 and Cancer irroratus Say, 1817. Can. Tech. Rept. Fish. aguat. Sci. (1403): 1-20.

ElneR, R.W. \& R.E. Lavole. 1983. Predation on American oysters (Crassostrea virginica (Gmelin)) by American lobsters (Homarus americanus Say), and mud crabs (Neopanope sayi (Smith)). Jour. Shellfish Res. 3 (2): 129-134. 
HAEFNER, P.A. JR. 1990 Natural diet of Callinectes ornatus (Brachyura: Portunidae) in Bermuda. Jour. Crust. Biol. 10 (2): 236-246.

HYNES, H.B.N. 1950. The food of fresh-water sticklebacks (Gasterosteus aculeatus and Pygosteus pungitius) with a review of method used in studies of the food fishes. Jour. Anim. Ecol. 19 (1): 36-51.

KAWAKAMI, E. \& G. VAZzoler. 1980. Método gráfico e estimativa de índice alimentar aplicado no estudo de alimentação de peixes. Bolm Inst. Oceanogr., São Paulo, 29 (2): 205-207.

KNEIB, R.T. 1982. The effects of predation by wading birds (Ardeidae) and blue crab (Callinectes sapidus) on the population size structure of the common mummichog, Fundulus heteroclitus. Estuar. Coast. Shelf Sci. 14: 159-163.

LAUGHLIN, R.A. 1982. Feeding habits of the blue crab, Callinectes sapidus Rathbun, in the Apalachicola estuary, Florida. Bull. mar. Sci. 32: 807-822.

LaZARo-Chavez, C. R. E. \& F. Buckle-Ramirez. 1994. Feeding habits and food niche segregation of Callinectes sapidus, C. rathbunae, and C. similis in a subtropical coastal lagoon of the Gulf of Mexico. Jour. Crust. Biol. 14 (2): 371-382.

LE CREN, E.D. 1951. The length-weight relationship and seasonal cycle in gonad weight and condition. Anim. Ecol. 20 (27): 201-219.

LIPCIUS, R.N. \& A.H. HINES. 1986. Variable functional responses of a marine predator in dissimilar homogeneous microhabitats. Ecology 67 (5): 1361-1371.

LUDWIG, J.A. \& J.F. REYNOLDS. 1988. Statistical ecology: a primer on methods and computing. New York, John Wiley \& Sons, Inc., 338p.

MCLAughlin, P.A.\& J.F. HeBARD. 1961. Stomach contents of the Bering Sea King crab. Bull. Int. N. Pacif. Fish Commn 5: 5-8.

Melo, G.A.S.; V.G. Veloso \& M.C. Oliveira. 1989. A fauna de Brachyura (Crustacea, Decapoda) do litoral do Estado do Paraná. Lista preliminar. Nerítica, Pontal do Paraná, 4 (1/2): 1-31.

NonAto, E.F. \& A.C.Z. Amaral. 1979. Anelídeos poliquetas. Chaves para famílias e gêneros. São Paulo, 78p.

Nomato, E.F.; M.A.V. PetTi \& P.C. Paiva. 1990. Contribuição dos anelídeos poliquetas na dieta de crustáceos decápodas braquiúros da região de Ubatuba. In: Simpósio de ecossistemas da costa sul e sudeste brasileira, Águas de Lindóia, São Paulo. ACIESP 71 1: 224-234.

PAUL, R.K.G. 1981. Natural diet, feeding and predatory activity of the crabs Callinectes arcuatus and C. toxotes (Decapoda, Brachyura, Portunidae). Mar. Ecol. Prog. Ser. 6: 91-99.

RIos, E.C. 1975. Braziliam marine mollusks iconography. Rio Grande, Fund. Univ. Rio Grande, 1328p.

RoPES, J.W. 1968. The feeding habits of the green crab, Carcinus maenas (L.). Fishery Bull. Fish Wildl. Serv. U.S. 67 (2): 183-203.

SANTOS, E. P. DOS. 1978. Dinâmica de populações aplicada à pesca e piscicultura. São Paulo, HUCITEC/EDUSP, 129p.

SEED, R. 1980. Predatior-prey relationships between the mud crab Panopeus 
herbstii, the blue crab, Callinectes sapidus and the Atlantic ribbed mussel Geukensia(=Modiolus) demissa . Estuar. coast. mar. Sci. 11: 445-448

SEed, R.; M.N. ElLIOT; P.J.S. BOAdEN \& R.J. o’CONNOR. 1981. The composition and seasonal changes amongst the epifauna associated with Fucus serratus $\mathrm{L}$. in Strangford Lough, Northern Ireland. Cah. Biol. Mar. 22: 243-266.

Stevens, B.G.; D.A. Armstrong \& R. Cusimano. 1982. Feeding habits of the dungeness crab Cancer magister as determined by the index of relative importance. Mar. Biol. 72: 135-145.

StONER, A.W. \& B.A. BUCHANAN. 1990. Ontogeny and overlap in the diets of four tropical Callinectes species. Bull. Mar. Sci. 46 (1): 3-12.

TAISsoun, E.N. 1969. Las especies de cangrejos del genero "Callinectes" (Brachyura) en el Golfo de Venezuela e Lago Maracaibo. Bolm. Cent. Invest. biol. 2: $1-112$.

WEAR, R.G. \& M. HADDON. 1987. Natural diet of the crab Ovalipes catharus (Crustacea, Portunidae) around central and northen New Zealand. Mar. Ecol. Progr. Ser. 35: 39-49.

Williams, A.B. 1974. The swimming crabs of the genus Callinectes (Decapoda: Portunidae). Fish. Bull. 72 (3): 685-798. 1984. Shrimps, lobsters and crabs of the Atlantic coast of the Eastern United States, Maine to Florida. Washington, Smithsonian Institution Press, $550 \mathrm{p}$.

Williams, M.J. 1981. Methods for analysis of natural diet in portunid crabs. (Crustacea: Decapoda: Portunidae). Jour. expl mar. Biol. Ecol. 52: 103-113. - 1982. Natural food and feeding in the commercial sand crab Portunus pelagicus Linnaeus, 1766 (Crustacea: Decapoda: Portunidae) in Moreton Bay, Queensland. Jour. Exp. Mar. Biol. Ecol. 59: 165-176.

Recebido em 17.X.1996; aceito em 20.XII.1997. 